\title{
Effects of Pictures on the Organization and Recall of Social Information
}

\author{
Michael Lynn, Sharon Shavitt, and Thomas Ostrom, Ohio State University
}

The role of visual stimuli in the organization and recall of social information was investigated in a study that presented photographs of stimulus persons along with verbal trait descriptors. Paired with four trait descriptors of each stimulus person, subjects saw either (a) no picture, (b) one trait-unrelated picture, (c) four trait-unrelated pictures, or (d) four traitrelated pictures. These conditions permitted a test of several competing explanations for the previously obtained improvement in memory for semantic information when accompanied by pictorial information. Results indicated that pictures incremented recall of trait information in two distinct stages-once with the addition of pictorial information and again when the pictures became relevant to the traits. Clustering in free recall on the basis of person categories was unaffected by the experimental conditions. These findings were consistent with the hypothesis that pictures enhance person memory by fostering elaboration on stimulus information at encoding.

Experimental research in cognitive psychology has clearly established that verbal material is remembered better if it is associated with visual information of some kind (Bower, 1972; Kosslyn \& Pomerantz, 1977; Madigan, 1983; Paivio, 1979). Most of this research, however, has employed relatively simple, uninvolving stimuli. Recently, Swann and Miller (1982) provided data indicating that visual information can enhance memory for more complex social stimuli as well. They found that videotapes of stimulus persons increased subjects' recognition of information about those persons. However, this pictorial enhancement of memory was observed only for vivid imagers' recognition of highly imageable information.

In discussing their results, Swann and Miller (1982) suggested that visual information might increase person memory in any of three ways: by strengthening person organization of social information, by serving as highly memorable cues to retrieval, or by motivating more elaborate processing of the material. Analogous explanations for this effect have arisen in the area of consumer behavior (Childers \& Houston, 1984). In this article, we formulate each of these three explanations in 
terms of associative network theory (e.g., J. R. Anderson, 1977; J. R. Anderson \& Bower, 1973) and report a study that evaluates the relative accuracy of their predictions. This will help to identify the cognitive processes through which visual information improves social memory.

The study employed a within-subjects design consisting of four levels of pictorial accompaniment to person information. In each experimental condition, subjects were presented four trait descriptors (one at a time) for each of four different persons. The trait descriptors of each person in the four experimental conditions were accompanied by either no photograph, the same trait-unrelated photograph, four different trait-unrelated photographs, or four different trait-related photographs. The trait-unrelated photographs were head and shoulder shots of the stimulus persons who displayed essentially neutral facial expressions. The trait-related photographs depicted the stimulus persons in poses and actions consistent with the traits they accompanied. After seeing each group of stimulus items, subjects free recalled the trait descriptors. Total recall and person clustering in recall were the principal dependent measures.

\section{Theoretical Models}

We have attempted to use the general concepts of associative network theory to develop

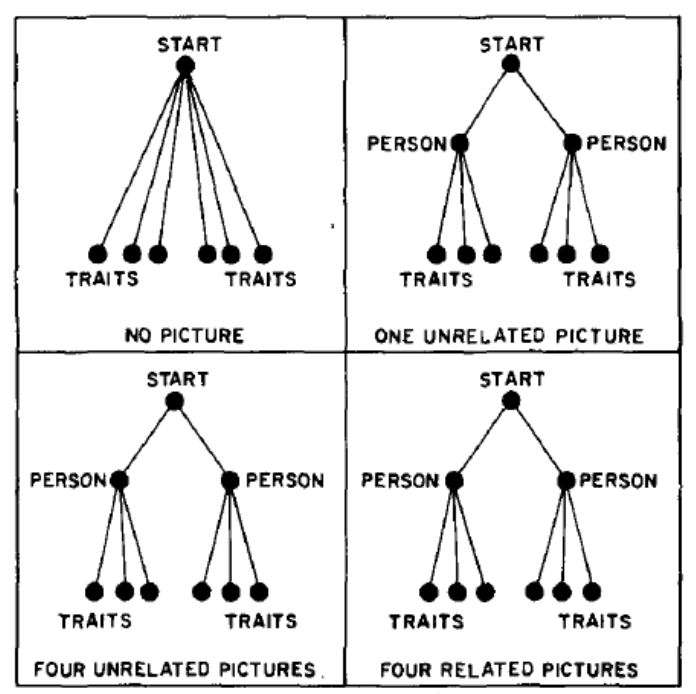

Figure 1. Associative network representation of the enhanced person organization model in which pictures increase the salience of persons as organizing categories. models that apply Swann and Miller's (1982)

explanations of picture effects on person memory to the experiment previously outlined. In these models, person names, traits, and pictures are all represented as nodes in memory. The three explanations offered by Swann and Miller (1982) suggest that subjects will form different patterns of associative relations among these memory nodes and that they will ultimately produce different patterns of recall and person clustering in our study. 
Our three models are illustrated with associative network diagrams in Figures 1-4. Although the experimental stimulus groups contained four facts about four persons, the figures (for simplicity) show three facts about two persons. As mentioned earlier, person names, traits, and pictures are depicted as nodes in memory. Associations between the nodes are shown as lines; this lines denote weaker associations and thick lines denote stronger associations. $^{1}$

In our models, the retrieval process is assumed to begin at a node representing "things learned in this experiment" (labeled start in our

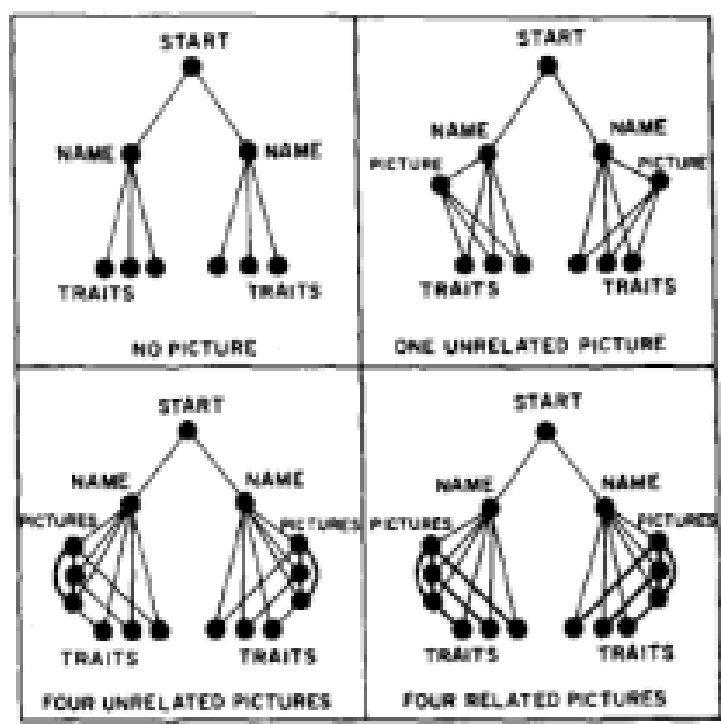

Figure 2. Associative network representation of the enhanced person organization model in which pictures increase the number and strength of intrapersonal associative pathways. diagrams) and to proceed serially along associative pathways to other nodes in memory. After a trait node is activated, the memory search is continued until another trait node is reached. When several associative pathways emanate from an activated node, the direction of the search is assumed to follow the strongest associative pathway. If all pathways are equally strong, then the subsequent path is chosen at random. This process is assumed to continue until either all the trait items are recalled or until one trait node has been activated several times, convincing subjects that a further search will result in no additional recall.

Given these assumed retrieval processes, the associative pathways between nodes should influence recall and person clustering according to the following principles. Recall of an item should be more likely (a) the greater the number of pathways to it, (b) the greater the strength of these pathways, and $(c)$ the fewer the alternative pathways emanating from its access nodes. Person clustering in recall should be determined by the ratios of the number and strengths of intracategory to intercategory associations: The more and the stronger the within-persons pathways (relative to pathways between persons) are the more subjects should cluster by person categories.

\footnotetext{
${ }^{1}$ The associations between nodes represented in Figures 1-4 are the critical associations that must be assumed to exist if the corresponding model is to explain picture effects on memory. Of course, other associations, besides the ones depicted in the figures, may be formed. For example, direct pathways between pairs of traits will most certainly be formed, as will direct pathways from start to the traits. However, those are assumed to be constant across the four experimental conditions in this study and thus do not affect our predictions. They are left out of the figures for the sake of simplicity.
} 


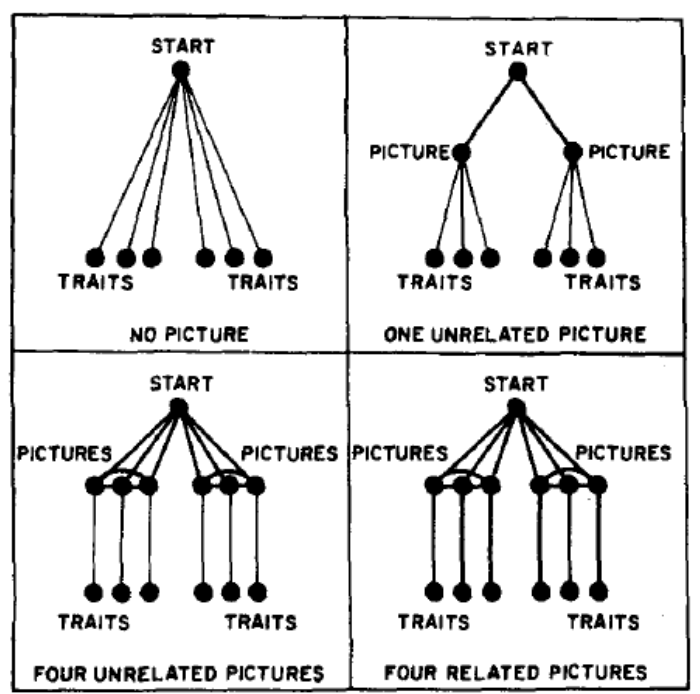

Figure 3. Associative network representation of the memnrahle retrieval cue model

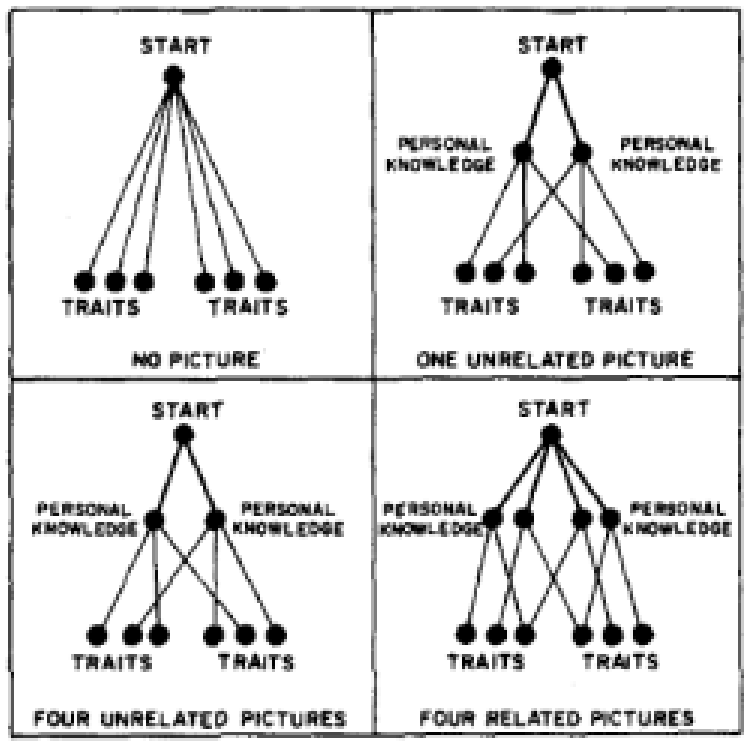

Figure 4. Associative network representation of the cognitive elaboration model.
The associative network diagrams in Figures 1 and 2 depict the enhanced person organization explanation for picture effects on memory. According to this explanation, pictures improve recall' by increasing the number of intrapersonal associative pathways in memory. This implies that the addition of pictures should increase person clustering in free recall.

There are at least two ways in which pictures could increase the number of intrapersonal associative pathways formed in subjects' memories. First, pictures could make persons more salient categories for organizing social information, and the enhanced salience of this organization category might lead subjects to encode the traits with the person names with which they were presented. In this case, there should be greater recall and person clustering with the introduction of pictorial information because persons serve as cues to retrieve the trait information (see Figure 1). There are no a priori reasons for believing that the number or relevance of the pictures would increase the salience of person categories. This explanation, then, predicts an increment in recall and person clustering when pictorial information is introduced but predicts no further increments with the introduction of multiple pictures or

trait-relevant pictures.

The second way in which pictures could increase the number of intrapersonal associations assumes that subjects encode the traits under person categories in all conditions. Subjects may associate pictures with both the verbal information they accompany and (when presented) with the other pictures of the same stimulus person. If so, then the number of intrapersonal associative pathways should increase as the number of pictures in each condition increases (see Figure 2). These more numerous intrapersonal associations mean that recall level and person clustering in recall should 
be higher in the four-trait-unrelated-pictures condition than in the one-trait-unrelated- picture condition.

According to this version of the model, recall and person clustering should also increase with the relevance of the pictures. Although the number of intrapersonal associative pathways is not higher in the four trait-related pictures condition than in the four trait-unrelated pictures condition, the strength of the pathways from the pictures to the traits increases. These stronger linkages would be expected to increase recall, and because they increase the strength of associations within each stimulus person, they would also be expected to increase person clustering.

The associative network diagrams in Figure 3 depict the memorable retrieval cue explanation for picture effects on person memory. According to this model, subjects use the pictures as retrieval cues for the trait information. As the number of pictures increases, so does the number of potential retrieval cues available to subjects. As the number of retrieval cues used by subjects increases, the number of alternative pathways from each retrieval cue to the trait information decreases. Because memory for an item of information is assumed to increase as the number of alternative pathways from its access nodes decreases, recall for the trait information should increase with the number of pictures. The four-traitrelated-pictures condition should produce the greatest recall. This is because, in addition to having only one retrieval cue per trait, the trait-related pictures carry information that is redundant with the verbal trait information. This should produce stronger associations between retrieval cues and traits.

The recall predictions of the memorable retrieval cue hypothesis coincide with those of the second version of the person organization hypothesis. However, the hypotheses differ in terms of their predictions regarding the pattern of person clustering in free recall. According to the memorable retrieval cue hypothesis, traits should be recalled together to the extent that they share a common retrieval cue or to the extent that their different retrieval cues are associated in memory. In the nopictures condition, traits describing different persons are accessed from the same node, implying that only chance levels of person clustering will occur. In the one-trait-unrelated-picture condition, all trait descriptions of the same person are accompanied by the same picture, or retrieval cue, so strong person clustering should occur. In the four-trait-unrelated- and the four-trait- related-pictures conditions, each trait is associated with a different picture of that person. Those pictures of the same person, however, should be associated with one another in memory due to their similarity. Therefore, person clustering should occur in these conditions at a level comparable to that in the one trait-unrelated picture condition. 
The associative network diagrams in Figure 4 depict a cognitive elaboration explanation of picture effects on person memory (see Craik \& Tulving, 1975, for a discussion of the effects of encoding elaboration on recall). Subjects' cognitive elaboration of the stimulus material is assumed to increase with the amount and complexity of visual information provided in each condition. The more visual information received, the more connections subjects are likely to draw between the stimulus material and themselves, other situations they have experienced, and other persons they know (see the cognitive response approach advocated by Petty, Ostrom, \& Brock, 1981). Pictures may enhance this elaboration because their complexity and richness activate more existing memory nodes to which the stimulus material can be related and/or because their vividness increases subjects' involvement and motivation to process the information elaborately. Either way, subjects' idiosyncratic knowledge categories serve as cues to retrieval and should enhance memory for the stimulus material.

This model clearly predicts that recall should be higher in the one-trait-unrelated-picture condition than in the no-picture condition and that it should be higher in the four-trait- related-pictures condition than in the four-trait-unrelated-pictures condition. There is some ambiguity, however, in predicting the effects of going from one to four trait-unrelated pictures. Because the pictures used in these conditions are similar to one another (i.e., all neutral head and shoulder shots), it is possible that little or no additional cognitive elaboration will occur by increasing their number from one to four. If this is true, no increase in recall would occur between these two conditions. Figure 4 was diagramed to reflect this possibility.

Little or no person clustering should occur in any of the experimental conditions under the cognitive elaboration model. This is because subjects are linking the stimulus traits to their own knowledge structures rather than to stimulus person categories. There is no rea son to expect that subjects' idiosyncratic categories will coincide with the arbitrary assignment of stimulus traits to stimulus persons.

\section{Method}

\section{Overview}

This study used a within-subjects design consisting of four levels of one factor: presence and type of pictorial information. Stimulus sets in the form of decks of cards with person-trait information were created to correspond to each of these four conditions. After reading each of the decks, subjects 
free recalled the trait descriptors they had been presented. Recall level, person clustering in free recall, and errors in recall were the dependent measures.

Subjects

Thirty-two undergraduate students from Ohio State University introductory psychology classes participated in this study in partial fulfillment of a course requirement. Each subject was run individually after being randomly assigned to one of the four counterbalanced orders of the conditions.

\section{Stimulus Materials}

The stimulus materials for this study comprised four sets of 64 cards containing semantic (trait) and pictorial information about 16 persons. Each set of 64 cards was divided into four decks containing four descriptors of each of four persons (two men and two women). Each deck of cards contained one of four different levels of pictorial information for each stimulus person: One deck contained no pictures; one contained four copies of the same trait-unrelated picture; one contained four different traitunrelated pictures, and one contained four different trait-related pictures. The order of these four different picture conditions was counterbalanced in a Latin square design; 8 subjects participated in each counterbalanced order. The semantic information on these cards was in the same order in every set of cards, so that counterbalancing the order of the picture conditions also counterbalanced the pairing of the picture conditions with the semantic information.

The semantic (name-trait) information items were put onto index cards in sentence form, and the descriptors were underlined (e.g., "Barb is studious"). Care was taken to ensure that (in the authors 'judgments) no two traits on the same continuum (e.g., conservative and liberal) or with the same meaning (e.g., good-natured and cheerful) appeared within the same deck. The trait descriptors were chosen so that a photograph of a single person performing an act or striking a pose could adequately depict the trait. Some of these traits were obtained from N. H. Anderson's (1968) list of rated adjectives, and the rest were generated by the authors.

Black and white photographs served as the visual stimuli for this study. Sixteen persons each posed for five trait-unrelated pictures and four trait-related pictures. In the trait-unrelated pictures, our models were photographed in standing and sitting postures in front of a variety of different backgrounds. In these pictures, however, our models displayed essentially neutral expressions and were engaged in no particular activity. Thus, subjects viewing these photographs only received information about the stimulus persons' appearance. In the four trait-related pictures, the models performed acts or 
assumed facial expressions that depicted the trait descriptors on the cards. For example, the photograph accompanying "Laura is tidy" showed a woman dusting a shelf. Thus, the visual information in these pictures corresponded to the accompanying semantic information.

\section{Procedure}

Subjects were told the experiment concerned memory for person information. After completing a practice trial that involved states and their capitals, subjects progressed through the experiment as follows: Each card in a stimulus deck was held up by the experimenter while the subjects read it aloud. Subjects were given $3 \mathrm{~s}$ to look at the card after reading it. The experimenter then put the card face down and held up the next one. This continued until all the cards in the deck were presented.

After presentation of each deck, subjects were given a distractor task of counting backward by 3 $s$ from a random three-digit number. Subjects were then asked to recall as many of the traits as they could and to write them down on a response sheet in the order that they came to mind. ${ }^{2}$ After the final recall trial, subjects were debriefed and thanked for their participation.

\section{Dependent Measures}

The measure of person clustering used in this study was the Adjusted Ratio of Clustering (ARC) index (Roencker, Thompson, \& Brown, 1971) advocated by Ostrom, Pryor, and Simpson (1981). It is based on the sequence in which items are listed in free recall. A category repetition is denned as two items from the same category (i.e., a person) appearing contiguously. The ARC score represents the proportion of category repetitions above chance obtained in free recall out of the total number of category repetitions above chance possible in the protocol. An ARC score of 1.00 indicates perfect clustering by that category system (persons); a score of zero indicates chance clustering, and a negative ARC score indicates that a subject is using some strategy that interferes with clustering by persons. Three person repetitions were built into the information order within each deck so that ARC would equal zero, given perfect serial recall of all items.

The dependent measure of recall consisted of the number of correctly recalled traits. A trait response was scored as correct if it was the same (regardless of spelling) as the trait word used in the

\footnotetext{
${ }^{2}$ Different subjects were unintentionally given different time limits on the recall task. One experimenter (who ran 6 subjects) allowed an unlimited time for recall and three other experimenters permitted a maximum of 2 min on each recall task. The patterns of means were similar for both groups of experimenters, however, and two analyses of variance with time limit as a between-subjects factor and picture condition as a within-subjects factor revealed no significant interaction effect on either total recall, $7-1(3,90)=0.48$, ns, or person clustering, $F(3,90)=0.04, n s$.
} 
stimulus deck. Synonyms were scored as errors in recall, as were words recalled from previous decks and words that did not appear in any of the decks. ${ }^{3}$

\section{Results}

Each dependent measure was subjected to a two-way analysis of variance (ANOVA) with picture condition as a within-subjects factor and counterbalance order as a between-subjects factor.

Recall Scores

Overall, the mean number of traits recalled was 6.94 out of a possible 16 (see Table 1). An ANOVA revealed that recall differed significantly among picture conditions, $F(3,84)=13.59, p<.0001$, but not among counterbalancing orders, $\mathrm{F}(3,28)=0.28$, ns, or the Picture Condition X Counterbalancing Order interaction, $F(9,84)=1.73$, ns. Post hoc pair-wise comparisons (using Fisher's least significant difference tests) between the four picture conditions yielded significant ( $\mathrm{ps}<.05$ ) differences between all conditions except the one- and four-trait- unrelated-pictures conditions. Thus, recall was increased by pictures in two distinct stages. The first stage occurred with the inclusion of some (vs. no) pictorial information, and the second stage occurred when the pictures became relevant to the trait information.

\section{Clustering Scores}

The mean person ARC score over all conditions was .14 (see Table 1), which was significantly different from zero, $F(1,28)=11.00$, $p<.003$. An ANOVA revealed no main effect for picture condition, $\mathrm{F}(3,84)=0.11$, ns, or counterbalancing order, $F(3,28)=0.83$, ns; but did show a significant Picture Condition $\mathrm{X}$ Counterbalance Order interaction, $F(9,84)=2.55$, $p<.02$. This interaction appeared to result from an increase in subjects' person clustering across trials. Latin square counterbalancing allows a test

Table 1 Mean Recall and Adjusted Ratio of Clustering (ARC) by Person Scores in Each of the Four Picture Conditions

\begin{tabular}{llc}
\hline \multicolumn{1}{c}{ Condition } & Recall & $\begin{array}{c}\text { ARC } \\
\text { by person }\end{array}$ \\
\hline $\begin{array}{l}\text { No picture } \\
\text { One trait-unrelated } \\
\text { picture }\end{array}$ & $5.78_{\mathrm{a}}$ & .14 \\
$\begin{array}{l}\text { Four trait-unrelated } \\
\text { pictures }\end{array}$ & $6.81_{\mathrm{b}}$ & .16 \\
$\begin{array}{l}\text { Four trait-related } \\
\text { pictures }\end{array}$ & $6.69_{\mathrm{b}}$ & .15 \\
Grand $M$ & $8.47_{\mathrm{c}}$ & .09 \\
\hline
\end{tabular}

Note. Scores with different subscripts are significantly $(p<.05)$ different from one another.

\footnotetext{
${ }^{3} 3$ Assuming prepositional representation in memory properly requires that synonyms be accepted as correctly recalled items. However, the use of personality traits made it difficult to determine whether or not words were synonyms of the stimulus traits. To avoid this difficult and subjective decision task, only the exact stimulus words were counted as correct.
} 
of the trial main effect. A significant linear trend was found across trials, $F(l, 28)=9.05, p<.01$. The mean of person ARC scores increased from Trial 1 to Trial 4: $-.02, .07, .16$, and .34.

\section{Error Scores}

Subjects made an average of 0.77 errors in each condition. This value did not differ significantly among the four picture conditions, $F(3,84)=1.45$, ns, or among the counterbalancing orders, $F(3,28)=$ 0.41 , ns. Furthermore, the interaction of these two factors was not significant, $F(9,84)=0.99$, ns.

\section{Discussion}

\section{Summary of Results and Their Relation to Previous Findings}

Our results support and extend Swann and Miller's (1982) findings. Like they did, we found that pictures enhanced subjects' memories for previously unfamiliar, hypothetical stimulus persons. Furthermore, because the trait-related pictures in our study should have increased the imageability of the traits in question, the fact that these pictures produced the highest recall is somewhat consistent with Swann and Miller's finding that pictures enhanced memory only for easily imageable information. Inconsistent with Swann and Miller, however, we found that pictures in- creased memory even when the imageability of the traits was not facilitated -that is, when traits were accompanied by unrelated pictures. In addition, we obtained our results with an unselected subject sample; Swann and Miller found memory enhancement only with vivid imagers.

One possible explanation for the apparently stronger effects of pictures on memory in this study compared with Swann and Miller's is that the two studies employed different dependent measures. Swann and Miller used a recognition measure of memory, and the present study employed a free-recall measure. It is generally accepted that recall involves two processes: the generation of potential targets and the recognition of the correct target (Crowder, 1976). If, as the results of this study suggest, pictures increase elaboration in encoding of social information, then they should enhance item generation but should not affect item recognition. Thus, Swann and Miller's dependent measure might have underestimated the impact of visual information on person memory processes.

Person clustering in free recall was not affected by the presence or absence of different types of pictures. On the other hand, a significant but low level of person clustering was obtained on an experiment wide basis $(M=.14)$. This low level of person clustering is consistent with previous research 
(Ostrom et al., 1981; Pryor \& Ostrom, 1981; Pryor, Simpson, Mitchell, Ostrom, \& Lydon, 1982) that has found that information about unfamiliar persons is not always clustered around persons in free recall.

Although person clustering was not affected by the picture conditions, it did increase as subjects acquired more experience with the learning task. Person clustering started at -.02 in Trial 1 and reached .34 by Trial 4 . On the first trial, subjects would have no reason to anticipate the dominant categorical structure inherent in the stimulus ensemble. As they learned this structure (i.e., the presence of person categories) over trials, they began adopting it as the basis for encoding the information. Apparently, this tendency was independent of the cognitive elaboration processes introduced by the picture conditions.

The fact that pictures did affect person memory but did not affect person clustering in free recall is diagnostic. Both the enhanced person organization and the memorable retrieval cue explanations for picture effects on person memory predict condition differences on person clustering. Consequently, these results suggest that some other mechanism underlies the effects of visual stimuli on memory for social information. One such explanation is that pictures enhanced subjects' elaboration of the material during encoding. The cognitive elaboration model made no predictions about the effects on memory of increasing the number of similar trait-unrelated pictures. We found that recall was constant across the one-and four-trait-unrelated-pictures conditions. This suggests that increasing the number of similar trait-unrelated photos did not provide more material or incentive for elaboration in encoding the trait information.

\section{Bases for Idiosyncratic Cognitive Elaboration}

Our data do not reveal the nature of the cognitive elaboration underlying picture effects on memory. However, our clustering data do suggest that this elaboration is not stimulus person focused. The content of elaboration was apparently based on material from subjects' idiosyncratic knowledge categories, categories that did not coincide with our arbitrary assignment of stimulus traits to stimulus persons. In encoding the stimulus materials, subjects could have employed idiosyncratic knowledge structures dealing with their own selves, other people they know, generic person types, situations they have experienced, or implicit trait theories they hold.

Subjects' self-schemata, or knowledge structures about the self (cf. Markus, 1977), might have contributed to the cognitive elaboration of stimulus persons' traits. Self-schemata can provide a framework for the perception of other people, much as they affect the processing of self-relevant information (see Markus \& Smith, 1981, for a review). These self-structures guide the interpretation of stimuli by focusing attention on domains that are relevant to the perceiver's self-concept. Thus, as 
Markus and Smith (1981) noted, when some feature of a stimulus person is relevant to a domain that is important to the perceiver, this feature tends to be elaborated on with material from the perceiver's own self-schemata. This analysis suggests that as subjects in our study encoded the information about the stimulus persons, they might have focused on and elaborated on the traits that were relevant to their own self-schemata, relating those traits to their own personality, behaviors, and judgment criteria. Pictures might have facilitated this elaboration either by motivating more attention to the selfschematic relevance of the stimulus traits or by suggesting additional self-schematic domains to which subjects could link the traits.

Subjects could also have related stimulus trait information to other knowledge structures in a similar manner. Characteristics of the stimulus perons could have been related to characteristics of other people or situations with which the subjects were familiar. For example, a subject could have related traits from different stimulus persons to features describing a familiar person (Pryor \& Ostrom, 1981) or a person type (Brewer, Dull, \& Lui, 1981), using the characteristics of this person as a framework for encoding the stimulus material. Pictures may motivate, or provide additional material for, drawing such connections between the stimulus information and friends, acquaintances, famous persons, and experiences.

Subjects could also have been influenced by their implicit personality theories (Rosenberg \& Sedlak, 1972; Schneider, 1973) in processing the stimulus traits. Implicit personality theories (IPTs), a type of impression formation schema, represent perceivers' expectations about the normative cooccurrence of traits. For example, a subject whose IPT includes the assumption that the traits studious and tidy tend to co-occur might have linked stimulus person information about Barb (studious) with information about Laura (tidy) in memory. Pictures might have facilitated the activation of subjects' IPTs in one of the two manners suggested earlier: by motivating more attention to the notion that one's implicit expectations are relevant to the stimulus information or by providing additional attribute information that could activate subjects' IPTs.

The previous discussion is not intended as an exhaustive list of possible elaborative content used by subjects in encoding the stimulus material. Other types of knowledge structures might also have been employed, contributing additional types of elaborative content. Furthermore, there is no reason to believe that each subject used the same type of knowledge category as a framework for encoding. Some subjects might have linked stimulus information to their own IPTs, others to self-schematic domains, and so forth. Which type of knowledge structure was activated for a particular subject might have been 
a function of the match between the stimulus material and the content of his or her own idiosyncratic knowledge categories.

Picture-Relevance Effect

In this study, recall was highest in the trait-related picture condition. One possible reason for this picture-relevance effect is that the trait-related pictures might have led subjects to encode the trait information even more elaborately than they did in the trait-unrelated pictures conditions. The traitrelated pictures were richer and more complex stimuli than were the trait-unrelated pictures, and this added complexity could have increased subjects' elaboration on the trait information in any of the ways previously outlined. The diagram and discussion of the cognitive elaboration model presented in our introduction reflect this possibility.

Although the cognitive elaboration explanation of the picture-relevance effect on memory has the advantage of being consistent with our explanation of the picture effect on memory, there are alternative explanations that deserve mention. One possibility is that the trait-related pictures increased the imageability of the trait information and that this enhanced imageability improved recall. Another possibility is that the trait-related pictures served as redundant sources of the trait information and that this redundancy in stimulus presentation enhanced recall of the traits. Unfortunately, our study does not provide a means of testing these different explanations of the picture- relevance effects on memory. Such a test is left to future research.

\section{References}

Anderson, J. R. (1977). Memory for information about individuals. Memory \& Cognition, 5, 430-442.

Anderson, J. R., \& Bower, G. H. (1973). Human associative memory. Washington, DC: Winston.

Anderson, N. H. (1968). Likableness ratings of 555 personality- trait words. Journal of Personality and Social Psychology, 9, 272-279.

Bower, G. H. (1972). Mental imagery and associative learning. In L. Gregg (Ed.), Cognition in learning and memory (pp. 51-88). New York: Wiley.

Brewer, M. B., Dull, J., \& Lui, L. (1981). Perceptions of the elderly: Stereotypes as prototypes. Journal of Personality and Social Psychology, 41, 656-670.

Childers, T. J., \& Houston, M. J. (1984). Conditions for a picture-superiority effect on consumer memory. Journal of Consumer Research, 11, 643-654. 
Craik, F. I. M., \& Tulving, E. (1975). Depth of processing and the retention of words in episodic memory. Journal of Experimental Psychology: General, 104, 268-294.

Crowder, R. G. (1976). Principles of learning and memory. Hillsdale, NJ: Erlbaum.

Kosslyn, S. M., \& Pomerantz, J. R. (1977). Imagery, propositions, and the form of internal representations. Cognitive Psychology, 9, 52-76.

Madigan, S. (1983). Picture memory. In J. C. Yuille (Ed.), Imagery, memory, and cognition (pp. 65-89). Hillsdale, NJ: Erlbaum.

Markus, H. (1977). Self-schemata and processing information about the self. Journal of Personality and Social Psychology, 35, 63-78.

Markus, H., \& Smith, J. (1981). The influence of self-schemata on the perception of others. In N. Cantor \& J. Kihlstrom (Eds.), Personality, cognition, and social interaction (pp. 233-262). Hillsdale, NJ: Erlbaum.

Ostrom, T. M., Pryor, J. R., \& Simpson, D. D. (1981). The organization of social information. In E. Higgins, C. Herman, \& M. Zanna (Eds.), Social cognition: The Ontario symposium (pp. 3-38). Hillsdale, NJ: Erlbaum.

Paivio, A. (1979). Imagery and verbal processes. Hillsdale, NJ: Erlbaum.

Petty, R. E., Ostrom, T. M., \& Brock, T. C. (Eds.) (1981). Cognitive responses in persuasion. Hillsdale, NJ: Erlbaum.

Pryor, J. B., \& Ostrom, T. M. (1981). The cognitive organization of social information: A converging operations approach. Journal of Personality and Social Psychology, 41, 628-641.

Pryor, J. B., Simpson, D. D., Mitchell, M., Ostrom, T. M., \& Lydon, J. (1982). Structural selectivity in the retrieval of social information. Social Cognition, 1, 336-357.

Roencker, D. L., Thompson, C. P., \& Brown, S. C. (1971). Comparison of measures for the estimation of clustering in free recall. Psychological Bulletin, 76, 45-48.

Rosenberg, S., \& Sedlak, A. (1972). Structural representations of implicit personality theory. In L. Berkowitz (Ed.), Advances in experimental social psychology (Vol. 6, pp. 235-297). New York: Academic Press.

Schneider, D. J. (1973). Implicit personality theory: A review. Psychological Bulletin, 79, 294-309.

Swann, W. B., \& Miller, L. C. (1982). Why never forgetting a face matters: Visual imagery and social memory. Journal of Personality and Social Psychology, 43, 475-480. 